\title{
Tension pneumothorax after ultrasound guided internal jugular venous catheterization in an inadvertently endobronchially intubated patient with kyphosis
}

\author{
Jin-Kook Son, IL-Ok Lee, Myoung-Hoon Kong, Nan Sook Kim, and Sang Ho Lim \\ Department of Anesthesiology and Pain Medicine, Guro Hospital, Korea University College of Medicine, Seoul, Korea
}

Ultrasound guidance has been promoted as a means of reducing the risk of complications during central venous catheterization (CVC), and during internal jugular venous catheterization (IJV), and ultrasound guidance has been reported to reduce the risk of mechanical complications and catheterplacement failures and the time required for insertion [1].

Mild kyphosis or scoliosis does not change cardiopulmonary function, but more aggravated deformities of spine have detrimental effects [2]. Complex interactions during head and neck movement and the fixed insertion depths of preformed endotracheal tubes often cause inadvertent malposition and may result in accidental extubation or inadvertent endobronchial intubation [3]. We report the case of a 53-year-old female patient with kyphosis who was inadvertently endobronchially intubated and rapidly developed tension pneumothorax after ultrasound guided IJV cannulation.

A 53-year-old female patient (133 cm tall and $51.5 \mathrm{~kg}$ ) with a diagnosis of kyphoscoliosis was admitted for posterior correction and fusion. At age 20, she contracted tuberculosis of spine and the kyphoscoliosis subsequently progressed to a kyphosis angle of 90 degrees one year prior to admission. No abnormal chest X-ray, pulmonary function test, laboratory result, or electrocardiogram findings were evident. Lidocaine (40 mg) with $1 \%$ propofol (Aquafol ${ }^{\circledR}$, Daewonpharm, Seoul, Korea) and remifentanil (Ultiva ${ }^{\mathrm{TM}}$, GlaxoSmithKline, UK) (Pion TCI ${ }^{\circledR}$, Bionet, Seoul, Korea) were administered at target concentrations of $4 \mu \mathrm{g} / \mathrm{ml}$ and $3 \mathrm{ng} / \mathrm{ml}$, respectively for induction. After administering $45 \mathrm{mg}$ of rocuronium, the trachea was intubated with a $7.0 \mathrm{~mm}$ armored endotracheal tube, and before fixing the tube $20 \mathrm{~cm}$ to incisors, lung sounds, chest wall movement in both lung fields, and endotracheal tube depth were confirmed; initial peak inspiratory pressure (PIP) was $15 \pm 1 \mathrm{cmH}_{2} \mathrm{O}$. The neck area was prepared and a $7.5-\mathrm{MHz}$ linear-array ultrasound probe, which was connected to a realtime ultrasound unit (SonoSite, Bothell, USA). The probe was covered with ultrasonic gel, wrapped in a sterile plastic sheath and focused at a depth of $4.5 \mathrm{~cm}$.

Standard ultrasound two-dimensional (2D) imaging was used to measure the depth and caliber of the IJV, which was found to be patent, compressible, and without thrombi. Catheterization was performed with a 19-gauge, 10 -cm needle under continuous dynamic observation and real-time 2D images were obtained by placing the transducer parallel and superior to the clavicle, and over the groove between the sternal and clavicular heads of the sternocleidomastoid muscle. For left side CVC, the patient's head was rotated to the right, but several attempts at left IJV cannulation failed. During an attempt to catheterize the left subclavian vein, vital signs changed to blood pressure $65 / 47$ $\mathrm{mmHg}$, heart rate 70 beats/min, and oxygen saturation $84 \%$. We immediately converted to manual ventilation with $100 \% \mathrm{O}_{2}$, but severe resistance was experienced with a PIP $35 \pm 4 \mathrm{cmH}_{2} \mathrm{O}$. A chest radiograph revealed unilateral tension pneumothorax in right lung and right endobronchial intubation (Fig. 1). The patient's head was then rotated to the neutral position and the intubation tube was withdrawn to $17 \mathrm{~cm}$ from the incisors. Needle aspiration was immediately performed and a chest tube

Corresponding author: IL-Ok Lee, M.D., Ph.D., Department of Anesthesiology and Pain Medicine, Guro Hospital, Korea University College of Medicine, 97, Gurodong-gil, Guro-gu, Seoul 152-703, Korea. Tel: 82-2-2626-3234, Fax: 82-2-851-9897, E-mail: iloklee@korea.ac.kr (c) This is an open-access article distributed under the terms of the Creative Commons Attribution Non-Commercial License (http:// creativecommons.org/licenses/by-nc/3.0/), which permits unrestricted non-commercial use, distribution, and reproduction in any medium, provided the original work is properly cited. 


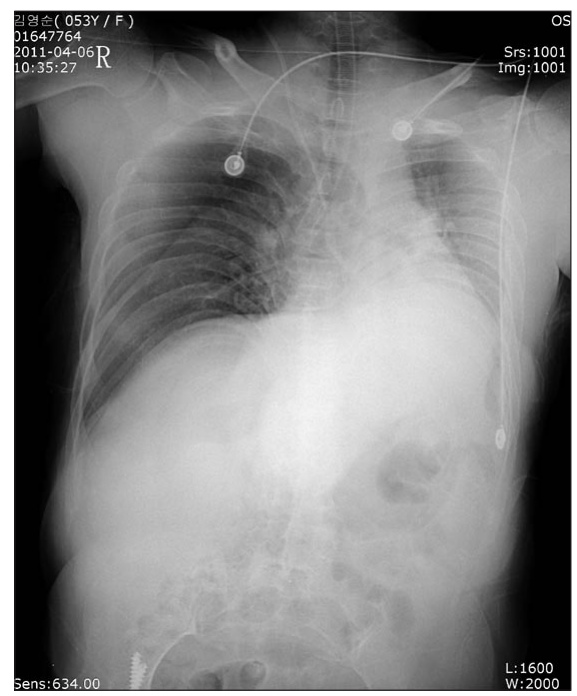

Fig. 1. Chest AP view showing right tension pneumothorax and endobronchial intubation.

was inserted into the thoracic cavity. Ventilation then improved and PIP was $24 \mathrm{cmH}_{2} \mathrm{O}$, and ABGA showed a pH 7.45, $\mathrm{PaCO}_{2} 35$ $\mathrm{mmHg}, \mathrm{PaO}_{2} 252 \mathrm{mmHg}$, and $\mathrm{SaO}_{2} 100 \%$. After the procedure had been completed, the patient was transferred to the intensive care unit with intubation. The chest tube was removed and the patient was discharged without sequelae on the 21th POD.

The use of CVCs may be associated with adverse effects that are hazardous to patients, and these complications are increased by patient anatomy (morbid obesity, cachexia, or local scarring from surgery or radiation treatment), the patient setting (mechanical ventilation), and the operator's skill. In our opinion, the anatomical abnormality and operator inexperience probably contributed in this case.

Ultrasound guidance can be performed using either the in-plane (IP) or the out-of-plane (OOP) techniques. The IP technique is performed with the cannula along the long axis of the ultrasound probe, whereas the OOP technique is performed with the cannula along the short axis of the ultrasound probe, and both techniques have advantages and disadvantages. The advantage of the IP technique is that the entire needle (body and tip) is visible and its disadvantage is that reverberation artifacts impair visualization of structures behind the needle. In particular, in cases with a large needle-to-probe distance the needle may be completely obscured. The OOP technique has the advantage that the needle tip is easier to visualize (some block techniques more useful with the OOP technique [4]), and that the needle causes no significant artifacts, and its disadvantage is that the body of the needle is not visualized, which means that if the correct plane of the body of the needle is lost, the body of the needle may be mistaken for the tip. We performed IJV cannulation using the OOP technique in the described case, and we believe that inadvertent misleading introduction of needle was a possible reason of this complication.

The diagnosis of pneumothorax may be difficult when manifestations are non-specific, but early diagnosis and treatment are important to prevent tension pneumothorax. Even though the rate of pneumothorax following real-time ultrasound-guided IJV puncture is extremely low, careful consideration of operator, technical, and patient factors may help to reduce the risk of pneumothorax [4].

The kyphosis angle of our patient was 90 degrees, and severe chest wall deformity, a narrow thoracic inlet, and inadequate positioning of the patient secondary to contractures contributed to the difficulty of achieving CVC [5], which demonstrates that ultrasound-guided CVC is not a fail-safe technique in terms of avoiding pneumothorax. Chest wall deformity due to kyphosis also contributed to the risk of endotracheal tube malposition when the head and neck were rotated. We advise that anesthesiologists should be aware of the occurrence of pneumothorax during ultrasound-guided central venous catheterization and of endotracheal tube malposition due to head and neck movement in kyphosis.

\section{References}

1. Oner B, Karam AR, Surapaneni P, Phillips DA. Pneumothorax Following Ultrasound-Guided Jugular Vein Puncture for Central Venous Access in Interventional Radiology: 4 Years of Experience. J Intensive Care Med 2011 [Epub ahead of print].

2. Stoelting RK, Diredorf SF. Anesthesia and co-existing disease. 4th ed. Philadelphia, Churchill Livingstone. 2002, pp 534-35.

3. Yap SJ, Morris RW, Pybus DA. Alterations in endotracheal tube position during general anaesthesia. Anaesth Intensive Care 1994; 22: 586-8.

4. Bhatia A, Lai J, Chan VW, Brull R. Case report: pneumothorax as a complication of the ultrasound-guided supraclavicular approach for brachial plexus block. Anesth Analg 2010; 111: 817-9.

5. Leung S, Malhotra AD, Eisen LA. Vascular access challenge on a patient with cerebral palsy and severe kyphoscoliosis. J Vasc Access 2010; 11 : 66-8. 